\title{
Energy Aware Data Collection with Route Planning for 6G Enabled UAV Communication
}

\author{
Mesfer Al Duhayyim ${ }^{1}$, Marwa Obayya ${ }^{2}$, Fahd N. Al-Wesabi ${ }^{3,4}$, Anwer Mustafa Hilal ${ }^{5, *}$, \\ Mohammed Rizwanullah ${ }^{5}$ and Majdy M. Eltahir ${ }^{6}$
}

\author{
${ }^{1}$ Department of Natural and Applied Sciences, College of Community-Aflaj, Prince Sattam bin Abdulaziz University, \\ Saudi Arabia \\ ${ }^{2}$ Department of Biomedical Engineering, College of Engineering, Princess Nourah bint Abdulrahman University, \\ Saudi Arabia \\ ${ }^{3}$ Department of Computer Science, King Khalid University, Muhayel Aseer, Saudi Arabia \\ ${ }^{4}$ Faculty of Computer and IT, Sana'a University, Sana'a, Yemen \\ ${ }^{5}$ Department of Computer and Self Development, Preparatory Year Deanship, Prince Sattam bin Abdulaziz University, \\ AlKharj, Saudi Arabia \\ ${ }^{6}$ Department of Information Systems, King Khalid University, Muhayel Aseer, Saudi Arabia \\ ${ }^{*}$ Corresponding Author: Anwer Mustafa Hilal. Email: a.hilal@psau.edu.sa \\ Received: 04 July 2021; Accepted: 27 August 2021
}

\begin{abstract}
With technological advancements in $6 \mathrm{G}$ and Internet of Things (IoT), the incorporation of Unmanned Aerial Vehicles (UAVs) and cellular networks has become a hot research topic. At present, the proficient evolution of $6 \mathrm{G}$ networks allows the UAVs to offer cost-effective and timely solutions for real-time applications such as medicine, tracking, surveillance, etc. Energy efficiency, data collection, and route planning are crucial processes to improve the network communication. These processes are highly difficult owing to high mobility, presence of non-stationary links, dynamic topology, and energy-restricted UAVs. With this motivation, the current research paper presents a novel Energy Aware Data Collection with Routing Planning for 6G-enabled UAV communication (EADCRP-6G) technique. The goal of the proposed EADCRP-6G technique is to conduct energy-efficient cluster-based data collection and optimal route planning for $6 \mathrm{G}$-enabled UAV networks. EADCRP-6G technique deploys Improved Red Deer Algorithm-based Clustering (IRDAC) technique to elect an optimal set of Cluster Heads $(\mathrm{CH})$ and organize these clusters. Besides, Artificial Fish Swarm-based Route Planning (AFSRP) technique is applied to choose an optimum set of routes for UAV communication in $6 \mathrm{G}$ networks. In order to validated whether the proposed EADCRP-6G technique enhances the performance, a series of simulations was performed and the outcomes were investigated under different dimensions. The experimental results showcase that the proposed model outperformed all other existing models under different evaluation parameters.
\end{abstract}

Keywords: Unmanned aerial vehicle; $6 \mathrm{G}$ networks; artificial intelligence; energy efficiency; clustering; route planning; data collection; metaheuristics

This work is licensed under a Creative Commons Attribution 4.0 International License, which permits unrestricted use, distribution, and reproduction in any medium, provided the original work is properly cited. 


\section{Introduction}

In recent times, with the evolution of Internet of Things (IoT) and 6G technologies [1], the incorporation of cellular systems and UAVs has to shift towards novel network advance trends. At present, UAVs experience a rapid growth in different business applications. It is anticipated that these UAVs bring major advantages to its users in several areas like emergency communications, smart city construction, agriculture, mineral exploration, disaster assessment forestry, plant protection, power and oil pipeline inspections and other such broad application prospects [2]. Altogether, 6G UAV network can optimally fulfil a number of IoT applications and pave way to provide advanced services. In UAVs, the cellular converged network can perform as an aerial Access Node (AP)/Base Station (BS) to collect data from a huge amount of IoT nodes allocated to a specific extent and realize the connections with $6 \mathrm{G}$ networks. UAVs has the potential to interact with ground cellular BS. However, it creates a self-organizing cluster network via remote intelligence control framework [3]. Many UAVs are armed with distinct IoT devices to collect IoT data from distinct places simultaneously and send the collected data directly to the adjacent ground BSs or interact with ground BS via leader UAV with the help of AP ability. Though UAV flight and IoT device heavily rely upon power supply, all these devices have limited energy that severely influences the popularization and promotion of UAV-based IoT applications [4]. Thus, the collaborative process of multiple UAVs is a major problem to be used in radio spectrum resources. Further, it is essential to perform an advanced study on energy saving technology and spectrum usage under the basis of inexpensive hardware.

Transmission is a key aspect in a number of UAV schemes [5]. However, such UAV schemes have certain aspects of FANET such as high mobility of UAV nodes, sparse placement, and constraints in the available battery resource which altogether can hinder the stability and efficient transmission among the UAVs [6]. The high mobility nature of the UAV causes periodic changes in the topology. This requires a recurrent communication of new location among UAV nodes which results in energy utilization on the basis of overheads [7]. Comparatively, the sparse location of UAVs requires high energy for communication, owing to a considerably-longer distance among the UAVs. Smart clustering technique can enact the main role to enhance the energy efficiency of a network. Clustering is a process that splits the UAV nodes under many groups, when these nodes are located in a geographical place with certain similarities in achieving a particular purpose. In a cluster, every node is chosen as Cluster Head $(\mathrm{CH})$. A CH node is permitted through coordination tasks and is relied upon the data of its cluster member to its end [8,9].

The current research work presents a novel Energy Aware Data Collection with Route Planning for 6G-enabled UAV communication (EADCRP-6G) technique. The goal of the proposed EADCRP-6G technique is to perform energy-efficient cluster-based data collection and optimal route planning for $6 \mathrm{G}$-enabled UAV networks. The proposed EADCRP-6G technique includes an Improved Red Deer Algorithm-based Clustering (IRDAC) technique to elect a set of optimal Cluster Heads $(\mathrm{CH})$ and organize the selected clusters. Besides, Artificial Fish Swarm-based Route Planning (AFSRP) technique is deployed in the selection of optimum set of routes for UAV communication in $6 \mathrm{G}$ networks. In order to examine the enhanced performance of EADCRP$6 \mathrm{G}$ technique, a series of simulations was conducted and the outcomes were investigated under different dimensions.

\section{Related Works}

Liu et al. [10] proposed a novel non-stationary multi-mobility UAV-to-ship channel method. This method consists of SB and LoS modules as a result of sea water fluctuations. The MB 
component is presented based on the waveguide effects on sea surface. In the presented method, UAV is denoted by transmitter (Tx), ship is denoted by receiver ( $R x)$, and the clusters between $\mathrm{Rx}$ and Tx can be viewed as moving in random directions and velocities. Later, a few common statistical properties of the projected UAV-to-ship channel module was investigated and the results were derived. Spyridis et al. [11] aimed at tracking a mobile IoT device of unknown position with the help of a set of UAVs, armed with RSSI sensors. The DL method executed the clustering process in UAV network at standard interval on the basis of GCN framework. In this execution, the data regarding UAV and RSSI locations was used. A heuristic technique was employed to define the number of clusters in a dynamic manner at all the instances. While the partitions were defined by optimizing RSSI loss function.

Bushnaq et al. [12] presented a relative analyses of U-UAV and T-UAV-aided cellular traffic offloading from a geographical location that endures heavy traffic situations. With the help of stochastic geometry tools, the researchers derived a joint distance distribution among UAV, hotspot users, and TBS. Na et al. [13] proposed a synergetic system for sub-slot allocation and UAV trajectory planning. Since the developed problems suffered from complications and nonconvexity, an effective and iterative method was proposed to overcome the issues. Later, for fixed sub-slot duration and clustering state, the researchers optimized the UAV trajectory. In FarajiBiregani et al. [14], a method was proposed to efficiently maintain the clusters that approached RF source while at the same time, the remaining UAVs were eliminated and returned to the base. Qi et al. [15] proposed an improved hierarchical 6G IoT network using UAVs in sky and armed IRS. The scheme utilized BackCom to transmit the data in free ride method. Through beamforming process, IRS improves the energy of reflectable signals, thus enhancing the performance and distance of BackCom. Tang et al. [16] studied about battery-constrained FEEL. In this study, the UAVs had the potential to alter the frequencies of functioning CPU, extend the lifetime of batteries and evade early dropping from FL training. The researchers optimized the scheme by jointly assigning both wireless bandwidth and computation resources upon time-differing platforms based on DDPG-based approach. In this approach, a linear integration of latency and energy utilization was executed to estimate the system cost.

Li et al. [17] investigated a joint downlink transmission power control and trajectory strategy challenge in multi type UABSs transmission networks. In order to satisfy the signal to noise power ratio of the user including interference, every UABS should alter its transmission power and location. Based on the communications among different transmission connections, a non-cooperative MFTG was presented earlier to model the joint optimization problem. Later, a Nash equilibrium solution was resolved using two phases: firstly, a user in the provided area is clustered to attain the early placement of UABSs; next, MFQ learning method is projected to resolve the distinct MFTG problems. Kurdi et al. [18] proposed a bacteria-inspired heuristic algorithm for effective distribution of tasks between the placed UAVs. The utilization of multi-UAVs is a significant model to combat the distribution of RPW in palm plantations. In order to improve the energy consumption and cost-aware transmission, a novel method named MRDSASL was presented in $6 \mathrm{G}$ network [19]. Then, multi-variate regression function was utilized as a threshold to analyze the evaluated node condition. Lastly, soft step activation function identified the effective nodes via regression analyses. Based on the results from deep analyses, $6 \mathrm{G}$ framework was implemented through effective nodes. 


\section{The Proposed EADCRP-6G Model}

The proposed EADCRP-6G technique is designed to establish effective data collection and communication process in $6 \mathrm{G}$-enabled UAV networks. The proposed EADCRP-6G technique encompasses two major processes namely, IRDAC technique for cluster construction and AFSRP technique for route planning. The detailed working stages of these two processes are offered in subsequent sections.

\subsection{System Model}

The upcoming 6G IoT demands the expansion of communication coverage while this ultrahigh coverage is set to turn inevitable trends in the growth of smart cities. At present, drones are the most effectual solutions to improve transmission coverage due to time consumption and heavy cost incurred upon installing new BS. There are two major variances exist i.e., UAV communications and conventional ground wireless transmission. Here, UAV is loaded with fullycontrollable maneuverability upon three dimensions. The horizontal position and height of the UAV can be adjusted at any point of time for the optimization of transmission efficiency with ground node. As displayed in Fig. 1, cylindrical antenna array is utilized improve the strength of transmission connection in an efficient manner. In order to ensure transmission performance between the UAV and BS, BS should execute precise beam-forming and take a certain location of UAV. In current research work, it is assumed that GPS and inertial measurement units are given to motion sensing and position of the UAVs. Each UAV is aware of its own location, location of the ground station, and location of other neighboring UAVs. Also, UAVs are armed with long- and short-range transmission with the help of $6 \mathrm{G}$ technologies. While the former one is utilized for data transmission within the cluster in the name of intra-cluster transmission, the latter one is utilized for data transmission between ground station and the CHs. Moreover, UAV-based mobile transmission aims at explicit data rate $R_{b}$ with Quadrature Phase Shift Keying (QPSK) and predetermined modulation method.

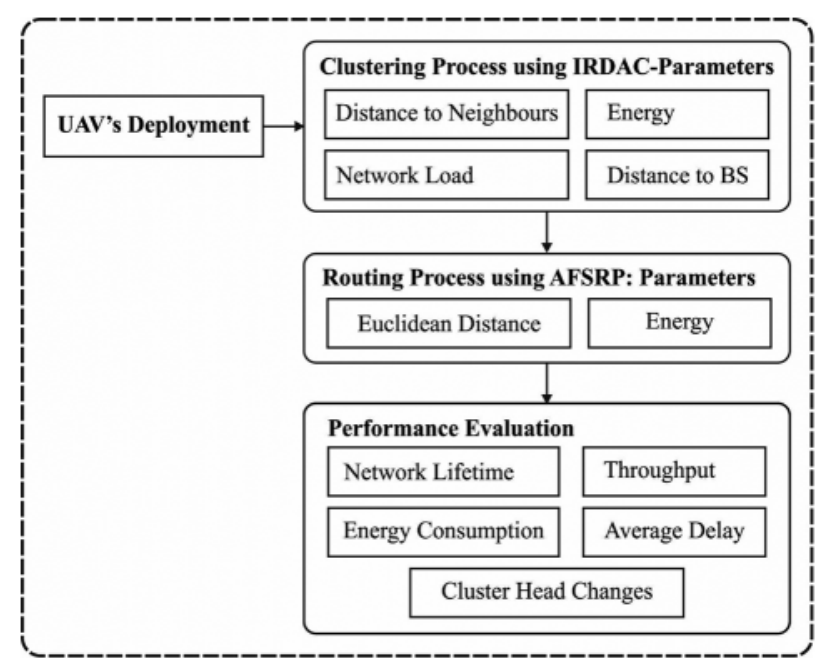

Figure 1: Overall working process of EADCRP-6G model 


\subsection{Design of IRDAC Technique}

RDA technique is initiated by an initial arbitrary population that is equivalent to that of the Red Deers (RDs). The number of optimum RDs in a population is determined and are named after 'male RD' while rest are named as 'hinds'. At first, male RD must roar. Depending upon the roaring capability, it is categorized under two groups such as Stags and Commanders. Alternatively, the commander builds the harem. Thus, the number of hinds in a harem is directly related with the capacity of commander in terms of fighting and roaring activities. At last, the commander mates with the hind in harem.

It is obvious that other males are named after stags for mating with adjacent hinds and no deliberation of constrained harems. Generally, the earlier steps of RDA evolve on the basis of exploration and exploitation phases efficiently. It can be stated that an RD provides a likely solution $\mathrm{X}$ in solution spaces. The dimension of solution $\mathrm{X}$ is Nvar. Succeeded by, Nvar dimension optimization problem, RD, is an $1 \times$ Nvar array that is formalized in Eq. (1):

RedDeer $=\left[X 1, X 2, X 3, \ldots, X_{N_{V A}}\right]$.

Indeed, Eq. (1) shows the element of $\mathrm{X}$. As well, the function value is evaluated for each RD in Eq. (2):

Value $=f($ RedDeer $)=f\left(X 1, X 2, X 3, \ldots, X_{N_{\text {var }}}\right)$.

To invoke this approach, the early population of size Npop is generated. Later, a group of optimum RD to Nmale is selected and the remaining one is Nhind (Nhind $=N p o p-$ Nmale). Thus, the Nmale count illustrates an elitist state of a method. In other perceptions, the number of Nmale maintains the increased features, whereas Nhind considers the diversification stage of the technique.

Now, a male RD attempts to improve the efficacy using roaring. Therefore, during roaring procedure, it may be ineffective/effective. It can be stated that a male RD is deliberated as an optimum solution. In the event of solution area, male $R D$ candidate is recognized. Apparently, it allows the male RD to alter its position. To upgrade the position of males, the expression given below is employed i.e., Eq. (3):

male $_{\text {new }}=\left\{\begin{array}{l}\text { male }_{o 1 d}+a_{1} \times\left((U B-L B) * a_{2}\right)+L \text { Bifa }_{3} \geq 0.5 \\ \text { male }_{o 1 d}-a_{1} \times\left((U B-L B) * a_{2}\right)+L B i f a_{3}<0.5\end{array}\right\}$

In spite of generating a likely neighborhood solution of males, UB and LB limit the search space. They are deliberated as UB and LB of the search space, consistently.

It is noted that male ${ }_{01 d}$ denotes the present location of male $R D$ whereas male $_{\text {new }}$ indicates the updated location. The randomization of roaring procedure, $a_{1}, a_{2}$ and $a_{3}$ are created arbitrarily by uniform distribution between $0 \& 1$. In order to develop a connection between this function and the roaring procedure, it can be observed that a male RD roars before it tries to expand the area. But, it stirs arbitrarily. These two conditions depend upon the solution space to demonstrate the influence of roaring procedure [20]. Further, $A \& B$ takes place normally in the roaring stage.

In fact, the dramatic variation exists between males i.e., $R D s$. Particularly, some are effective, energetic, and attractive in development compared to others. Therefore, RD is categorized as 
two components such as stags and commanders. The number of male commanders is defined by Eq. (4):

$N_{\text {Com }}=\operatorname{round}\left\{\gamma \cdot N_{\text {male }}\right\}$

Here, $N_{\text {Com }}$ denotes the count of males. It is indicated that $\gamma$ determines early value of a method. The range of numbers must be between zero \& one. Finally, the amount of stags are evaluated using Eq. (5):

$N_{\text {stag }}=N_{\text {male }}-N_{\text {Com }}$

whereas $N_{\text {stag }}$ denotes the amount of stags with respect to male population. In this method, RD population is used by including a number of hinds, commanders, and stags.

Consider that every commander fights with stags arbitrarily. Thus, two new solutions are obtained and exchanged by the commanders. These solutions are optimized whereas an optimal solution is superior to four solutions such as stag, commander, and two new solutions. In the event of fighting procedure, two mathematical formulas are used in Eqs. (6),(7):

$\left.N e w 1=\frac{(C o m+S t a g)}{2}+b_{1} \times\left((U B-L B) * b_{2}\right)+L B\right)$

$\left.N e w 2=\frac{(C o m+S t a g)}{2}-b_{1} \times\left((U B-L B) * b_{2}\right)+L B\right)$

Here, New1 \& New2 represent the new solutions created with the help of fighting. Stag and Com indicate stags and commanders, respectively. In the event of new solutions, UB and LB diminish the search spaces. Since the fighting procedure is randomized, $b_{1}$ and $b_{2}$ are created by uniform distribution function between 0 and 1. Consider four solutions like New2, Stag, Com, and New1 whereas OF is the optimum one. The connection and performance of male RDs is demonstrated in fighting. Thus, two new solutions are generated. One is determined as a winner whereas other one is a looser. As demonstrated, it contains two solutions. The OF solution for New1 is higher compared to another solution while New1 is the current commander. Fig. 2 illustrates the flowchart of RDA. Based on this procedure, harem is created. The amount of hinds in harem depends upon energy of male commanders. The efficacy of commander could be defined by $\mathrm{OF}$ amount. In order to develop the harems, hind is separated amongst the commanders by Eq. (8):

$y_{n}=v_{n}-\max \left\{v_{i}\right\}$

whereas $v_{n}$ denotes the energy of $\mathrm{n}^{\text {th }}$ commander and $y_{n}$ indicates the normal value. In order to estimate the normalized power of commander, the provided concept is utilized and is shown in Eq. (9).

$P_{n}=\left|\frac{V_{n}}{\sum_{\mathrm{i}=1}^{N_{C o m}} V_{i}}\right|$

Then, the number of hinds in a harem is calculated by Eq. (10):

$N \cdot$ harem $_{n}=\operatorname{round}\left\{P_{n} \cdot N_{\text {hind }}\right\}$ 
Here, N.harem $n$ denotes the number of hinds in $\mathrm{n}^{\text {th }}$ harem and Nhind displays the number of hinds. In order to classify the hinds, N.harem $n$ is chosen arbitrarily from hinds. It can be determined as shown in Eq. (11).

$N$. harem $_{n}^{\text {mate }}=$ round $\left\{\alpha . N\right.$. harem $\left._{n}\right\}$

Here, N.harem mate denotes the number of hinds in $\mathrm{n}^{\text {th }}$ harem that mates with the commander. About the solution space, N.harem mate of $N$. harem $_{n}$ is chosen arbitrarily.

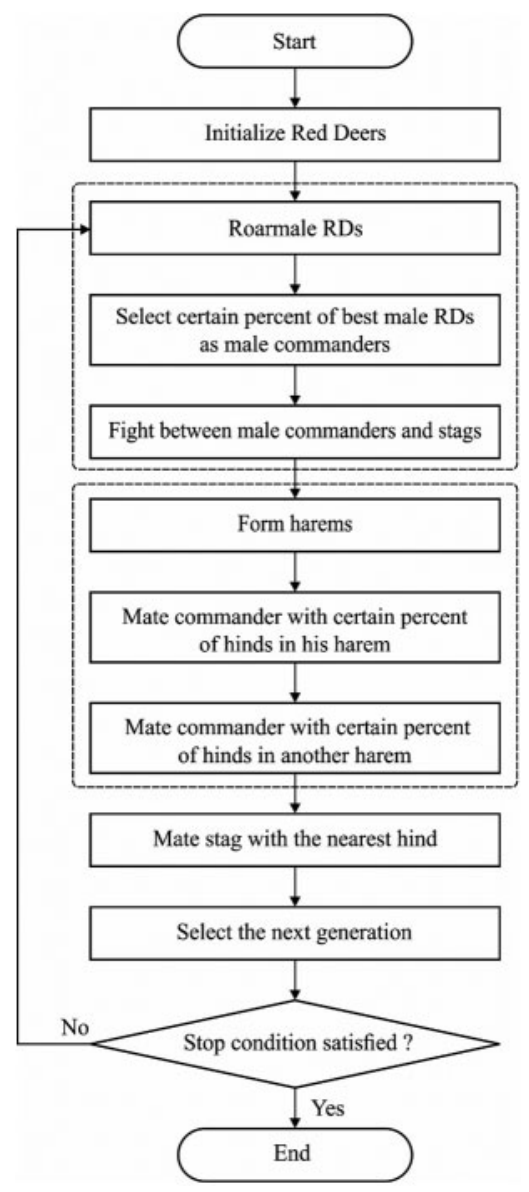

Figure 2: Flowchart of red deer algorithm

Succeeded by, $\alpha$ denotes the initial variable amount of RDA with values ranged between zero $\&$ one. Generally, mating procedure is defined as follows (12):

$o f f s=\frac{(\text { Com }+ \text { Hind })}{2}+(U B-L B) \times c$

Here, Com \& Hind denote hinds and commanders. Additionally, $c$ is created arbitrarily by a uniform distribution function from $0 \& 1$.

The harem is chosen in an arbitrary way and male commanders mate with $\beta$ number of hinds. Therefore, the commander attacks other harems to catch the opponent's region and increase the 
values. As well, $\beta$ displays a primary variable of this method. The amount of hinds in harem, mated the commander, is defined through the following Eq. (13):

$N$. harem $_{k}^{\text {mate }}=\operatorname{round}\left\{\beta . N\right.$. harem $\left._{k}\right\}$

whereas $N$.harem mate denotes the number of hinds in $\mathrm{k}^{\text {th }}$ harem that mates with the commander.

Now, the stags mate with nearby hind. During mating, a male RD needs to employ a useful hind. It is the desired hind among other hinds without supposing the harem region.

$d_{i}=\left(\sum_{j \in J}\left(\operatorname{stag} j-h i n d_{j}^{i}\right)^{2}\right)^{1 / 2}$

whereas $d_{i}$ denotes the distance from $\mathrm{i}^{\text {th }}$ hind and stag. It is chosen on the basis of two different standards. At first, each male RD is maintained to represent a commander and a stag. Next, it displays the remaining population in following generation. The generated offspring and hinds are chosen, based on the fitness value and by applying the roulette wheel/fitness tournament method. Since each approach is a well-known one, the interrelated mathematical equations are not provided. It can be included in huge iterations; the dominance of optimum solutions could be recognized within a certain period.

In order to eliminate the RDA from local optimal problem, Levy Distribution (LD) concept is incorporated in it. LD is an arithmetic method of initiating a sudden drift. Fig. 3 is a diagram of RDA module. Levy flight is an arbitrary walk procedure where the step length of searching tasks gets improved with a sudden drift and is denoted by.

$\operatorname{Levy}(\alpha) \sim t^{-1-\alpha}, \quad 0<\alpha<2$

whereas $t$ denotes a random variable in the range between zero and one and $\alpha$ represents the stability index. In LD scheme of the searching region, it could be given as follows.

$\operatorname{Levy}(\beta)=\frac{u \times \phi}{|v|^{1 / \beta}}$

whereas $u \& v$ denote the values of standard distribution, $\beta$ represents levy exponent and $\emptyset$ is given as follows

$\phi=\left[\frac{\Gamma(1+\alpha) \times \sin \left(\frac{\pi \alpha}{2}\right)}{\Gamma\left(\frac{(1+\alpha)}{2}\right) \times \alpha \times 2^{\left(\frac{\alpha-1}{2}\right)}}\right]^{\frac{1}{\alpha}}$

whereas $\alpha$ is equivalent to $1.5, u \& v$ represent random values. In IRDA method, a random individual is given the following equation to initiate the procedure of population.

$X_{i}= \begin{cases}X_{l b}+a \times \operatorname{Levy}(\alpha) \times\left(X_{j}-X_{k}\right) & \text { if } r_{1}()<r_{2}() \\ X_{u b}+a \times \operatorname{Levy}(\alpha) \times\left(X_{j}-X_{k}\right) & \text { otherwise }\end{cases}$

whereas $r()$ denotes a random variable. When RDA is not appropriately used in the search space, Levy imposed solution contributes to the unchanged feature of current individual. 


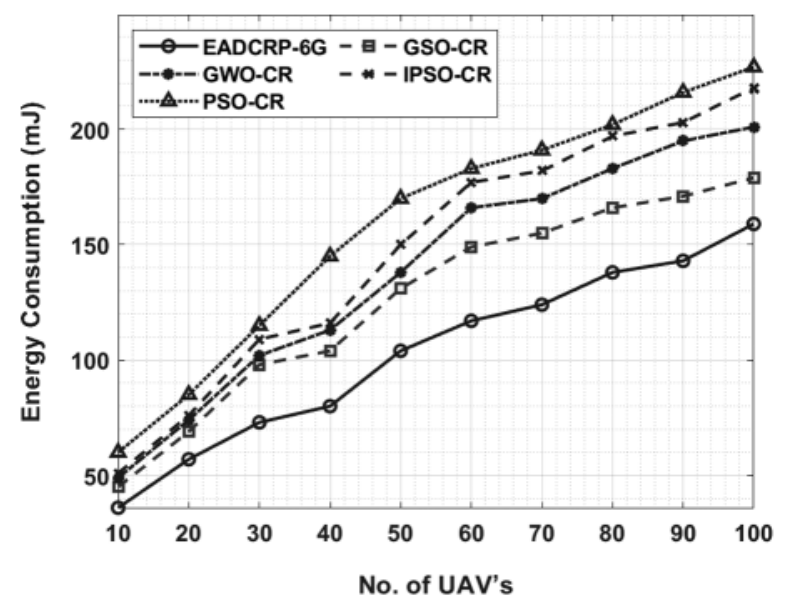

Figure 3: Energy consumption analysis of EADCRP-6G model

Generally, there exists four objective functions while every function encloses a substantial factor which is required for many purposes such as energy efficient clustering, energy maintenance by limiting the number of $\mathrm{CHs}$, recognizing the current energy ratio, reducing the distance between $\mathrm{BS}$ and $\mathrm{CH}$, mitigation of intracluster distances, enhanced intercluster distance between $\mathrm{CHs}$, and load balancing amongst CHs. The recently-proposed hybrid WGWO method provides an optimum solution by deriving a FF with the help of distance to neighbours, energy, network load, and distance to BS. Thus, FF is defined in the recently used method as follows.

$F F=\operatorname{minimum}\left(m_{1} y_{1}+m_{2} y_{2}+m_{3} y_{3}+m_{4} y_{4}\right)$

Here, $m_{1}, m_{2}, m_{3}$, and $\mathrm{m}_{4}$ denote the weight constants defined by user, and $F F$ indicates the FF. In addition, $y_{1}, y_{2}, y_{3}$, and $y_{4}$, indicate the objective functions. The proposed IRDA is employed in the identification of an optimum solution on the basis of FF. Now, the solution is attained by an agent. Therefore, the optimized solution for the aforementioned FF is made up of minimum number of clusters with maximal link $<$ quality and dynamically-determined CHs with maximum RE. The primary function is to conserve energy whereas energy ratio is defined below. When there are $R$ clusters and $M$ nodes, then the ratio of nodes' energy and current CHs energy are defined through Eq. (20).

$y_{1}=\frac{\sum_{p=1}^{M} \text { Energy }_{\left(\text {node }_{p}\right)}}{\left.\sum_{q=1}^{R} \text { Energy }_{\left(\text {cluster }_{\text {head }}\right)}\right)}$

The following function defines the distance to neighbours, defined as Euclidean distance between the SNs.

$y_{2}=\sum_{q=1}^{R} \frac{\sum_{\forall \operatorname{node}_{j} \in \operatorname{cluster}_{q}} \text { euclidean_distance }\left(\text { node }_{j}, \text { cluster_head }_{q}\right)}{\text { minimum }_{\forall \text { node }_{j} \in \text { clusterr }_{q}} \text { euclidean_distance }\left(\text { node }_{j}, \text { cluster_head }_{q}\right)}$ 
The $3^{\text {rd }}$ function decreases the distance between $\mathrm{CH} \& \mathrm{BS}$. This method is deliberated as $A \times A$ where the entire cluster denotes $R$ as demonstrated in Eq. (21).

$y_{3}=\frac{\left.\frac{1}{R} \sum_{q=1}^{R} \text { euclidean_distance (cluster_head }{ }_{q}, \text { base }- \text { station }\right)}{\frac{A}{2}}$

The last function is used for load management from CH. Eq. (23) tells the high load constraints amongst $\mathrm{CHs}$. $\left|C N_{q}\right|$ denotes the number of nodes in cluster $q$. Then, an area is deliberated as $A \times A$ while an entire cluster denotes $R$.

$y_{4}=\frac{\operatorname{MAXIMUM}\left(\left|C N_{q}\right|\right)}{\frac{1}{R} \sum_{q=1}^{R}\left(\left|C N_{q}\right|\right)}$

\subsection{Design of AFSRP Technique}

AFSA is a popular SI optimization module developed on the basis of fish swarming behaviour. This method is highly useful in the development of intelligent models to identify the global optimum solutions and does not attain gradient details of the objective function. Now, an artificial fish explores for food based on the foraging hierarchy of swarming nature and arbitrary behavior. Furthermore, artificial fish permits mutual data transmissions until a global optimal solution is attained. Assume $X=\left(x_{1}, x_{2}, \ldots x_{n}\right)$ as the location of artificial fish and $Y=f(X)$ represents the fitness at location $X$. Consider $d_{i j}=|| X_{i}-X_{j}||$ as the distance between the location $X_{i} \& X_{j}$, and Visual \& Step indicate the perception range and moving step of artificial fish, correspondingly.

(1) Foraging behavior

Assume $X_{i}$ as the present state of artificial fish and the position $X j$ is randomly selected from the Visual range. If $Y j<Y i$, then artificial fish is stirred up a Step in the way of $\left(X_{j}-X_{i}\right)$. Else, a position $X j$ is determined in an arbitrary manner to select before it encounters further conditions. If the criteria is not fulfilled, then a haphazard behavior is experienced. The foraging nature can be defined as follows.

$\tilde{X}_{i}= \begin{cases}X_{i}+\text { Step } \cdot \frac{X_{j}-X_{j}}{d_{j j}} \cdot \text { rand }, & \text { if }\left(Y_{j}<X_{i}\right) \\ \text { arbitarybehavior, } & \text { or else }\end{cases}$

whereas $\tilde{X}_{i}$ denotes the future position of artificial fish, rand represents the uniformly-generated values from zero and one.

(2) Swarming behavior

In a fish swarm, artificial fish $X_{i}$ has to search the intermediate position $X_{c}$ of $N_{F}$ artificial fish in current neighborhood $\left(d_{i j}<\right.$ Visual). When $\left(Y_{C} / N_{F}>\delta Y_{i}\right)$, the artificial fish $X_{i}$ gets stirred towards $X_{c}$. The numerical function of swarming behavior is given herewith.

$\tilde{X}_{i}= \begin{cases}X_{i}+\text { Step } \cdot \frac{X_{c}-X_{i}}{d_{i} C} \cdot \text { rand }, & \text { if }\left(Y_{c} / N_{F}<\delta \cdot Y_{i}\right) \\ \text { foragying behavior, } & \text { otherwise }\end{cases}$

whereas $\delta \in(0,1)$ represents food attentiveness.

(3) Following behavior 
When $X_{\text {lbest }}$ is a local optimal unit in present neighborhood of $X_{i}$, then, $\left(Y_{\text {lbest }} / N_{F}>\delta Y_{i}\right)$, the artificial fish $X_{i}$ gets stirred in a direction $\left(X_{\text {lbest }}-X_{i}\right)$. The arithmetical function of this behavior is given herewith.

$\tilde{X}_{i}= \begin{cases}X_{i}+\text { Step } \cdot \frac{X_{\text {lbest }}-X_{i}}{d_{i, l b e s t}} \cdot r \mathrm{cmd}, & \text { if }\left(Y_{\text {lbest }} / N_{F}<\delta \cdot Y_{i}\right) \\ \text { foragingbehavior, } & \text { otherwise }\end{cases}$

(4) Random behavior

The artificial fish determines a state arbitrarily from Visual range and travels to the corresponding location. It is called default behavior.

(5) Behavior selection

In the event of AF, the predetermined behavior is compared and performed [21]. Thus, an optimum nature is determined to upgrade the current position of AF. Bulleting can be employed to record the best position i.e., $X_{\text {best }}$ in a fish swarm. Each AF is related to respective position by bulletin after a step is forwarded. If the condition becomes normal, then a bulletin may get updated.

Thus, AFSA employs the social nature of fish swarm to resolve the optimization problem. It is highly helpful for fish environment data and self-information to change the search direction so as to gain an optimal convergence and diversity. Thus, AF attains a location, where the food resource is maximal. Although AFSA is a superior member of global optimization methods among other optimization problems, it is still at risk in converging to sub optimum methods like metaheuristics. It is called early convergence of complex optimization problems which results in low performance. In order to detect an optimum set of routes, the dimension of every fish is made equivalent to $\mathrm{CHs}$ and further positions are located in BS. Assume, $\theta^{i}=\left(\theta_{1}^{i}, \theta_{2}^{i} \mid \theta_{p+1}^{i}\right)$ denotes $i^{\text {th }}$ fish, $\theta_{n_{i}}^{i}$ denotes a real value in the range of zero and one. Then, the provided function is applied in the determination of next hop to BS and is determined as follows

$f(x)=\left\{i\right.$, for which $\left|\left(\frac{i}{k}-X_{i_{f} j}\right)\right|$ is minimum, $\forall_{i} 1 \leq i \leq k$

The aim is to define an optimal set of routes from $\mathrm{CH}$ to BS with the help of FF including two variables such as distance and energy. Initially, the RE of subsequent hop node is defined and the node with maximal energy is processed as a relay node. In order to transmit the data, the source node transmits it to relay node that additionally moves towards the BS through inter CHs. Thus, the node with high $\mathrm{RE}$ is processed as a subsequent hop node. The initial sub objective $f 1$ is given as herewith.

$f 1=\sum_{i=1}^{m} E_{C H i}$

Also, Euclidean distance is employed in the calculation of distance from $\mathrm{CHs}$ to $\mathrm{BS}$. The minimization of energy dissipation mostly depends upon the transmission distance. In the event of low distance, the energy gets considerably stored. When the distance is raised, a huge volume 
of energy is consumed. Hence, a node using minimal distance is preferred for a relay node. Thus, the following sub-objective i.e., $f 2$ through distance is denoted.

$f 2=\frac{1}{\sum_{i=1}^{\mathrm{m}} \operatorname{dis}\left(\mathrm{CH}_{i}, \mathrm{NH}\right)+\operatorname{dis}(\mathrm{NH}, \mathrm{BS})}$

The aforementioned sub-objectives are summarized as a FF herewith, in which $\alpha_{1}$ and $\alpha_{2}$ denote the weights allocated to every sub-objective.

Fitness $=\alpha_{1}(f 1)+\alpha_{2}(f 2)$, where $\sum_{i=1}^{2} \alpha_{i}=1 \alpha_{i} \varepsilon(0,1)$;

\section{Performance Validation}

The current section examines the performance of the proposed EADCRP-6G technique under different performance measures. Tab. 1 shows the results achieved from Energy Consumption Analysis (ECA) and Network Lifetime Analysis (NLTA) of EADCRP-6G technique under varying number of UAVs. Fig. 3 shows ECA results of the proposed EADCRP-6G technique against existing techniques under distinct UAVs. From the figure, it can be inferred that the proposed EADCRP-6G technique accomplished an optimum performance over other methods with low ECA. For instance, with 10 UAVs, EADCRP-6G technique obtained a minimal ECA of 36 mJ, whereas GSO-CR, GWO-CR, IPSO-CR, and PSO-CR techniques attained the maximum ECA values such as 45, 49, 51, and $60 \mathrm{~mJ}$ respectively. Meanwhile, with 30 UAVs, EADCRP6G approach attained a lesser ECA value of $73 \mathrm{~mJ}$, whereas GSO-CR, GWO-CR, IPSO-CR, and PSO-CR methods gained high ECA values of 98, 102, 109, and $115 \mathrm{~mJ}$ correspondingly. Eventually, with 50 UAVs, the proposed EADCRP-6G technique achieved a minimum ECA of $104 \mathrm{~mJ}$, whereas GSO-CR, GWO-CR, IPSO-CR, and PSO-CR techniques achieved high ECA values such as 131, 138, 150, and $170 \mathrm{~mJ}$ correspondingly. In line with these, at 70 UAVs, EADCRP-6G method obtained a minimal ECA of $124 \mathrm{~mJ}$, whereas GSO-CR, GWO-CR, IPSOCR, and PSO-CR methods gained maximum ECA values such as 155, 170, 182, and $191 \mathrm{~mJ}$ correspondingly. Lastly, with 100 UAVs, EADCRP-6G technique obtained the least ECA of 159 mJ, whereas GSO-CR, GWO-CR, IPSO-CR, and PSO-CR methodologies attained high ECA values such as $179,201,218$, and $227 \mathrm{~mJ}$ correspondingly.

Fig. 4 shows the detailed results of NLTA achieved by EADCRP-6G technique under varying UAVs. The experimental results demonstrate that the proposed EADCRP-6G technique attained effective outcomes with an improved NLTA. For instance, with 10 UAVs, EADCRP-6G technique demonstrated a high NLTA of 6250 rounds, whereas GSO-CR, GWO-CR, IPSO-CR, and PSOCR techniques produced low NLTA values such as 5900, 5850, 5660, and 5100 rounds respectively. Followed by, with 30 UAVs, the proposed EADCRP-6G approach outperformed other methods with a superior NLTA of 6000 rounds, while GSO-CR, GWO-CR, IPSO-CR, and PSO-CR methods yielded less NLTA values such as 5620, 5300, 5150, and 4920 rounds correspondingly. Concurrently, with 50 UAVs, EADCRP-6G technique exhibited a high NLTA of 5510 rounds, whereas GSO-CR, GWO-CR, IPSO-CR, and PSO-CR techniques achieved low NLTA values as given herewith; 5340, 5150, 4720, and 4370 rounds correspondingly. In the meantime, under 70 UAVs, EADCRP-6G method demonstrated a high NLTA of 5050 rounds, whereas GSO-CR, GWO-CR, IPSO-CR, and PSO-CR methods achieved low NLTA values such as 4740, 4790, 4140, and 4000 rounds correspondingly. Finally, under 100UAVs, the presented EADCRP-6G 
algorithm portrayed a maximum NLTA of 4490 rounds, whereas GSO-CR, GWO-CR, IPSO-CR, and PSO-CR methods achieved less NLTA values such as 3880, 3710, 3610, and 3540 rounds correspondingly.

Table 1: Results of the analysis of proposed EADCRP-6G method against existing methods with respect to energy consumption (mJ) and network lifetime (Rounds)

\begin{tabular}{llllll}
\hline No. of UAV's & \multicolumn{5}{l}{ Energy consumption $(\mathrm{mJ})$} \\
\cline { 2 - 6 } & EADCRP-6G & GSO-CR & GWO-CR & IPSO-CR & PSO-CR \\
\hline 10 & 36 & 45 & 49 & 51 & 60 \\
20 & 57 & 69 & 74 & 76 & 85 \\
30 & 73 & 98 & 102 & 109 & 115 \\
40 & 80 & 104 & 113 & 116 & 145 \\
50 & 104 & 131 & 138 & 150 & 170 \\
60 & 117 & 149 & 166 & 177 & 183 \\
70 & 124 & 155 & 170 & 182 & 191 \\
80 & 138 & 166 & 183 & 197 & 202 \\
90 & 143 & 171 & 195 & 203 & 216 \\
100 & 159 & 179 & 201 & 218 & 227 \\
\hline No. of UAV's & Network lifetime & Rounds) & & & \\
\cline { 2 - 6 } & EADCRP-6G & GSO-CR & GWO-CR & IPSO-CR & PSO-CR \\
\hline 10 & 6250 & 5900 & 5850 & 5660 & 5100 \\
20 & 6140 & 5850 & 5620 & 5480 & 5050 \\
30 & 6000 & 5620 & 5300 & 5150 & 4920 \\
40 & 5740 & 5580 & 5280 & 4930 & 4510 \\
50 & 5510 & 5340 & 5150 & 4720 & 4370 \\
60 & 5230 & 4950 & 5000 & 4410 & 4190 \\
70 & 5050 & 4740 & 4790 & 4140 & 4000 \\
80 & 4760 & 4410 & 4170 & 4090 & 3920 \\
90 & 4530 & 4130 & 3940 & 3700 & 3610 \\
100 & 4490 & 3880 & 3710 & 3610 & 3540 \\
\hline
\end{tabular}

Tab. 2 examines the results of throughput and average delay accomplished by the proposed EADCRP-6G method under varying numbers of UAVs. A brief throughput of EADCRP-6G method is shown in Fig. 5 under varying UAVs. The experimental outcomes showcase that the proposed EADCRP-6G method achieved effective outcomes with increased throughput. For sample, under $10 \mathrm{UAVs}$, the proposed EADCRP-6G technique displayed a superior throughput of $0.88 \mathrm{Mbps}$, whereas GSO-CR, GWO-CR, IPSO-CR, and PSO-CR algorithms offered low throughput values such as $0.82,0.81,0.79$, and $0.75 \mathrm{Mbps}$ correspondingly. Likewise, under $30 \mathrm{UAVs}$, the proposed EADCRP-6G technique demonstrated an increased throughput of 0.79 Mbps, whereas other methods such as GSO-CR, GWO-CR, IPSO-CR, and PSO-CR achieved low throughput values such as $0.69,0.61,0.56$, and $0.53 \mathrm{Mbps}$ respectively. Similarly, under 50 UAVs, EADCRP-6G method achieved a maximum throughput of $0.74 \mathrm{Mbps}$, whereas GSO-CR, 
GWO-CR, IPSO-CR, and PSO-CR techniques accomplished minimum throughput values such as $0.57,0.50,0.45$, and 0.37 Mbps respectively.

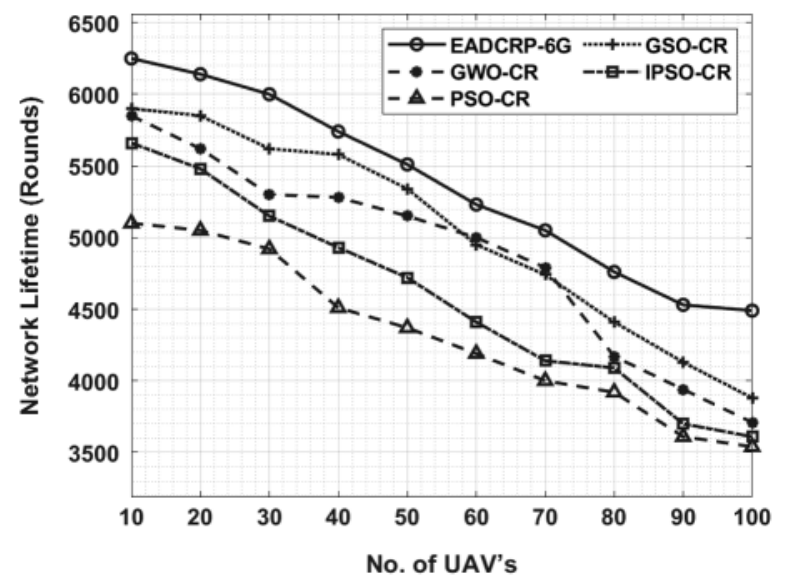

Figure 4: Network lifetime analysis of EADCRP-6G model

Table 2: Analysis results of the proposed EADCRP-6G technique against existing methods with respect to throughput (Mbps) and average delay (s)

\begin{tabular}{llllll}
\hline \multirow{2}{*}{ No. of UAV's } & \multicolumn{5}{l}{ Throughput (Mbps) } \\
\cline { 2 - 6 } & EADCRP-6G & GSO-CR & GWO-CR & IPSO-CR & PSO-CR \\
\hline 10 & 0.88 & 0.82 & 0.81 & 0.79 & 0.75 \\
20 & 0.85 & 0.76 & 0.70 & 0.66 & 0.62 \\
30 & 0.79 & 0.69 & 0.61 & 0.56 & 0.53 \\
40 & 0.76 & 0.61 & 0.55 & 0.49 & 0.42 \\
50 & 0.74 & 0.57 & 0.50 & 0.45 & 0.37 \\
60 & 0.72 & 0.53 & 0.47 & 0.43 & 0.34 \\
70 & 0.70 & 0.51 & 0.44 & 0.42 & 0.33 \\
80 & 0.67 & 0.50 & 0.42 & 0.41 & 0.31 \\
90 & 0.64 & 0.48 & 0.39 & 0.40 & 0.30 \\
100 & 0.63 & 0.46 & 0.38 & 0.37 & 0.28 \\
\hline No. of UAV's & Average delay (s) & & & & \\
\cline { 2 - 6 } & EADCRP-6G & GSO-CR & GWO-CR & IPSO-CR & PSO-CR \\
\hline 10 & 1.94 & 2.07 & 2.10 & 2.17 & 2.76 \\
20 & 2.06 & 2.57 & 2.85 & 3.67 & 4.86 \\
30 & 2.44 & 3.27 & 3.55 & 4.77 & 6.06 \\
40 & 3.66 & 4.17 & 4.75 & 5.57 & 7.76 \\
50 & 4.96 & 5.77 & 5.95 & 6.17 & 8.86 \\
\hline
\end{tabular}


Table 2: Continued

\begin{tabular}{llllll}
\hline No. of UAV's & \multicolumn{5}{l}{ Average delay (s) } \\
\cline { 2 - 6 } & EADCRP-6G & GSO-CR & GWO-CR & IPSO-CR & PSO-CR \\
\hline 60 & 5.79 & 6.25 & 6.65 & 6.76 & 9.25 \\
70 & 5.88 & 6.81 & 7.15 & 7.38 & 9.79 \\
80 & 6.33 & 7.07 & 7.72 & 8.26 & 10.29 \\
90 & 6.62 & 7.60 & 8.48 & 8.86 & 10.72 \\
100 & 6.88 & 8.06 & 8.82 & 9.09 & 11.79 \\
\hline
\end{tabular}

Also, under 70 UAVs, the proposed EADCRP-6G technique depicted an increase in the throughput of $0.70 \mathrm{Mbps}$, whereas GSO-CR, GWO-CR, IPSO-CR, and PSO-CR techniques achieved low throughput values such as $0.51,0.44,0.42$, and $0.33 \mathrm{Mbps}$ respectively. Lastly, under 100 UAVs, EADCRP-6G methodology outperformed all other methods with a superior throughput of $0.63 \mathrm{Mbps}$, whereas GSO-CR, GWO-CR, IPSO-CR, and PSO-CR methods achieved minimal throughput values such as $0.46,0.38,0.37$, and $0.28 \mathrm{Mbps}$ correspondingly.

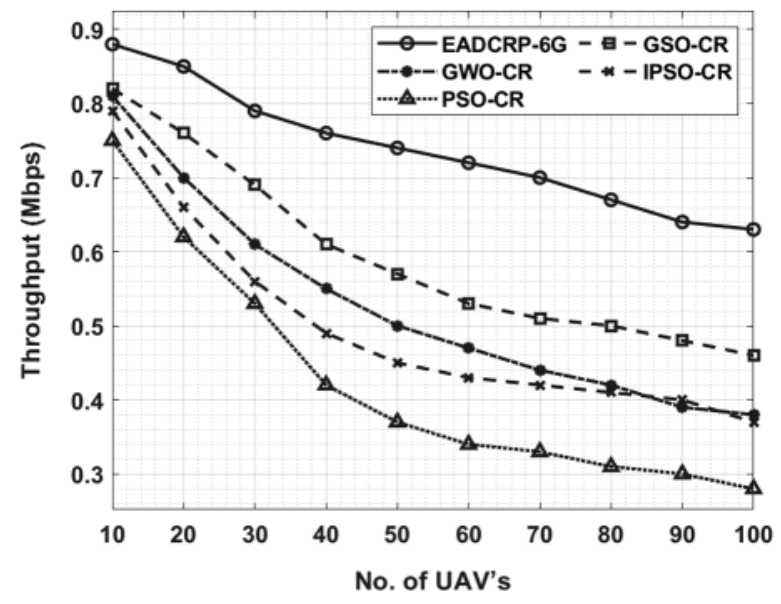

Figure 5: Throughput analysis of EADCRP-6G model

Fig. 6 shows the average delay results of EADCRP-6G method against existing algorithms under different UAVs. From the figure, it can be understood that the proposed EADCRP-6G technique accomplished a better performance over other methods with the least average delay. For instance, under $10 \mathrm{UAVs}$, the proposed EADCRP-6G method attained a low average delay of 1.94 s, whereas GSO-CR, GWO-CR, IPSO-CR, and PSO-CR methodologies reached maximal average delays of 2.07, 2.10, 2.17, and 2.76 s correspondingly. In line with this, at 30 UAVs, EADCRP-6G approach attained the minimum average delay of $2.44 \mathrm{~s}$, whereas GSO-CR, GWO-CR, IPSO-CR, and PSO-CR techniques attained maximum average delay values such as 3.27, 3.55, 4.77, and $6.06 \mathrm{~s}$ correspondingly. Likewise, with $50 \mathrm{UAVs}$, EADCRP-6G technique obtained a minimum average delay of $4.96 \mathrm{~s}$, whereas GSO-CR, GWO-CR, IPSO-CR, and PSO-CR techniques accomplished the maximum average delay time such as 5.77, 5.95, 6.17, and $8.86 \mathrm{~s}$ correspondingly. At the same time, with 70 UAVs, EADCRP-6G technique attained a less average delay of $5.88 \mathrm{~s}$, 
whereas GSO-CR, GWO-CR, IPSO-CR, and PSO-CR methods gained the maximum average delays such as $6.81,7.15,7.38$, and 9.79 s correspondingly. Finally, with 100 UAVs, the proposed EADCRP-6G manner attained the least average delay of $6.88 \mathrm{~s}$, whereas GSO-CR, GWO-CR, IPSO-CR, and PSO-CR algorithms attained the maximal average delay values such as $8.06,8.82$, 9.09 , and 11.79 s correspondingly.

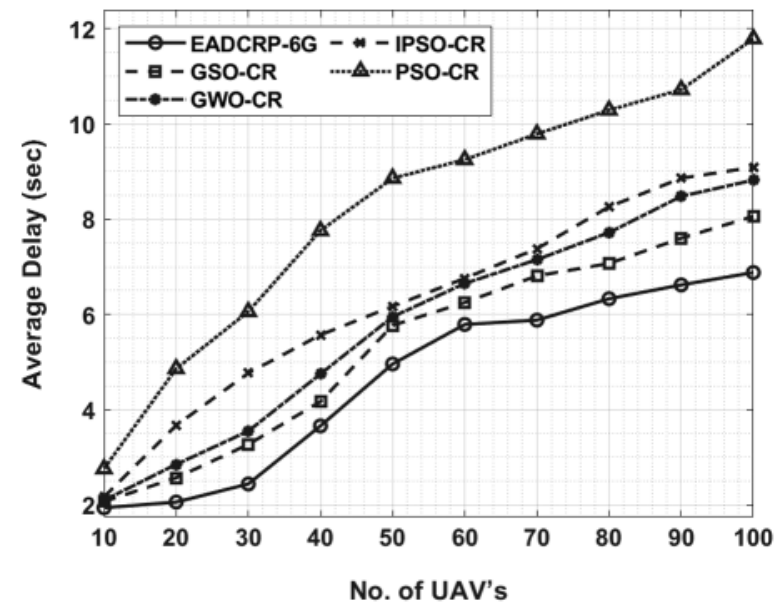

Figure 6: Average delay analysis of EADCRP-6G model

\section{Conclusion}

The current research paper presented a novel EADCRP-6G technique to execute effective data collection and communication process in 6G-enabled UAV networks. The proposed EADCRP-6G technique encompasses two major processes namely, IRDAC technique for cluster construction and AFSRP technique for route planning. EADCRP-6G technique includes IRDAC technique in order to elect an optimal set of $\mathrm{CHs}$ and organize the selected clusters. Besides, AFSRP technique is deployed to elect an optimum set of routes for UAV communication in 6G networks. In order to validate the enhanced performance of EADCRP-6G technique, a series of simulations was conducted and the outcomes were investigated under different dimensions. The experimental results showcase that the performance of the proposed model got enhanced under different evaluation parameters. In future, effective MAC scheduling techniques can be designed to accomplish effective resource-balanced 6G-enabled UAV networking.

Funding Statement: The authors extend their appreciation to the Deanship of Scientific Research at King Khalid University for funding this work under Grant Number (RGP 2/209/42), Received by Fahd N. Al-Wesabi. www.kku.edu.sa. This research was funded by the Deanship of Scientific Research at Princess Nourah bint Abdulrahman University through the Fast-Track Path of Research Funding Program.

Conflicts of Interest: The authors declare that they have no conflicts of interest to report regarding the present study. 


\section{References}

[1] F. Qi, W. Li, P. Yu, L. Feng and F. Zhou, "Deep learning-based BackCom multiple beamforming for 6G UAV IoT networks," EURASIP Journal on Wireless Communications and Networking, vol. 2021, no. 1, pp. 50, 2021.

[2] I. V. Pustokhina, D. A. Pustokhin, E. L. Lydia, M. Elhoseny and K. Shankar, "Energy efficient neurofuzzy cluster based topology construction with metaheuristic route planning algorithm for unmanned aerial vehicles," Computer Networks, vol. 196, pp. 108214, 2021.

[3] S. Sun, M. Kadoch, L. Gong and B. Rong, "Integrating network function virtualization with SDR and SDN for 4G/5G networks," IEEE Network, vol. 29, no. 3, pp. 54-59, 2015.

[4] I. V. Pustokhina, D. A. Pustokhin, P. K. Pareek, D. Gupta, A. Khanna et al., "Energy-efficient cluster-based unmanned aerial vehicle networks with deep learning-based scene classification model," International Journal of Communication Systems, vol. 34, no. 8, pp. 1-16, 2021.

[5] M. A. Khan, A. Safi, I. M. Qureshi and I. U. Khan, "Flying ad-hoc networks (FANETs): A review of communication architectures, and routing protocols," in 2017 First Int. Conf. on Latest trends in Electrical Engineering and Computing Technologies (INTELLECT), Karachi, pp. 1-9, 2017.

[6] A. Rajagopal, G. P. Joshi, A. Ramachandran, R. T. Subhalakshmi, M. Khari et al., "A deep learning model based on multi-objective particle swarm optimization for scene classification in unmanned aerial vehicles," IEEE Access, vol. 8, pp. 135383-135393, 2020.

[7] A. Khan, F. Aftab and Z. Zhang, "BICSF: Bio-inspired clustering scheme for FANETs," IEEE Access, vol. 7, pp. 31446-31456, 2019.

[8] A. Rajagopal, A. Ramachandran, K. Shankar, M. Khari, S. Jha et al., "Fine-tuned residual networkbased features with latent variable support vector machine-based optimal scene classification model for unmanned aerial vehicles," IEEE Access, vol. 8, pp. 118396-118404, 2020.

[9] B. L. Nguyen, E. L. Lydia, M. Elhoseny, I. V. Pustokhina, D. A. Pustokhin et al., "Privacy preserving blockchain technique to achieve secure and reliable sharing of IoT data," Computers, Materials \& Continua, vol. 65, no. 1, pp. 87-107, 2020.

[10] Y. Liu, C.-X. Wang, H. Chang, Y. He and J. Bian, "A novel non-stationary 6G UAV channel model for maritime communications," IEEE Journal on Selected Areas in Communications, vol. 37, pp. 1, 2021.

[11] Y. Spyridis, T. Lagkas, P. Sarigiannidis, V. Argyriou, A. Sarigiannidis et al., "Towards 6G IoT: Tracing mobile sensor nodes with deep learning clustering in UAV networks," Sensors, vol. 21, no. 11, pp. 3936, 2021.

[12] O. M. Bushnaq, M. A. Kishk, A. Celik, M.-S. Alouini and T. Y. Al-Naffouri, "Optimal deployment of tethered drones for maximum cellular coverage in user clusters," IEEE Transactions on Wireless Communications, vol. 20, no. 3, pp. 2092-2108, 2021.

[13] Z. Na, Y. Liu, J. Shi, C. Liu and Z. Gao, "UAV-supported clustered NOMA for 6G-enabled internet of things: Trajectory planning and resource allocation," IEEE Internet Things Journal, vol. 39, pp. 1, 2020.

[14] M. Faraji-Biregani and R. Fotohi, "Secure communication between UAVs using a method based on smart agents in unmanned aerial vehicles," The Journal of Supercomputing, vol. 77, no. 5, pp. 5076-5103, 2021.

[15] F. Qi, W. Li, P. Yu, L. Feng and F. Zhou, "Deep learning-based BackCom multiple beamforming for 6G UAV IoT networks," EURASIP Journal on Wireless Communications and Networking, vol. 2021, no. 1, pp. 50, 2021.

[16] S. Tang, W. Zhou, L. Chen, L. Lai, J. Xia et al., "Battery-constrained federated edge learning in UAVenabled IoT for B5G/6G networks," Physical Communication, vol. 47, pp. 101381, 2021.

[17] L. Li, Y. Sun, Q. Cheng, D. Wang, W. Lin et al., "Optimal trajectory and downlink power control for multi-type UAV aerial base stations," Chinese Journal of Aeronautics, vol. 34, pp. 11-23, 2021.

[18] H. Kurdi, M. F. AlDaood, S. Al-Megren, E. Aloboud, A. S. Aldawood et al., "Adaptive task allocation for multi-UAV systems based on bacteria foraging behaviour," Applied Soft Computing, vol. 83, no. 1, pp. 105643, 2019. 
[19] R. Sekaran, M. Ramachandran, R. Patan and F. Al-Turjman, "Multivariate regressive deep stochastic artificial learning for energy and cost efficient 6G communication," Sustainable Computing: Informatics and Systems, vol. 30, pp. 100522, 2021.

[20] G. N. Nguyen, N. H. L. Viet, A. F. S. Devaraj, R. Gobi and K. Shankar, "Blockchain enabled energy efficient red deer algorithm based clustering protocol for pervasive wireless sensor networks," Sustainable Computing: Informatics and Systems, vol. 28, pp. 100464, 2020.

[21] Q. He, X. Hu, H. Ren and H. Zhang, "A novel artificial fish swarm algorithm for solving large-scale reliability-redundancy application problem," ISA Transactions, vol. 59, no. 2, pp. 105-113, 2015. 